\title{
STABLE SUMMATION METHODS FOR A CLASS OF SINGULAR STURM-LIOUVILLE EXPANSIONS
}

\author{
HARVEY DIAMOND, MARK KON AND LOUISE RAPHAEL ${ }^{1}$
}

\begin{abstract}
Given the Sturm-Liouville eigenfunction expansion of an $L_{2}$ function $f(x)$, summability theory provides means for recovering the value of $f\left(x_{0}\right)$ at points $x_{0}$ where $f$ is sufficiently regular. If the coefficients in the expansion are perturbed slightly (in the $L_{2}$ norm), a stable summation method will recover from the perturbed expansion a good approximation to $f\left(x_{0}\right)$. In this paper we develop stable summation methods for expansions in eigenfunctions of the singular Sturm-Liouville system $u^{\prime \prime}-q(x) u=-\lambda u, u(0) \cos \beta+u^{\prime}(0) \sin \beta=0, u(\infty)<\infty$; where $q(x)$ $\in L_{1}[0, \infty)$ and continuous. Given a summability method known to work at $x_{0}$ for a particular expansion, our results say that if the summation parameter is appropriately scaled with the $L_{2}$ error in the perturbed expansion, a stable summation method is obtained. We obtain a sharp scaling requirement for guaranteeing stability. We apply our results to Riesz and Stieltjes summability.
\end{abstract}

1. Introduction and definitions. If we know that a Sturm-Liouville expansion is summable at a point (using a particular summation method), and we are given an approximation close in the mean square norm to the coefficients in that expansion, can we obtain a good approximation to the sum from the approximate expansion? The naive application of the summability method to the approximate expansion fails in general. If, however, the summation parameter is appropriately scaled with the mean square error, the desired stable approximation method can be obtained in many cases. Results of this nature are obtained in this paper for the case of a singular Sturm-Liouville system on a semi-infinite domain, possessing a discrete negative and continuous positive spectrum. The same Sturm-Liouville system was studied in [4] but results somewhat less powerful than ours were obtained and for one specific summability method only (see $\$ 3$ below). Other related results have been obtained for regular Sturm-Liouville systems [5] and for fundamental systems of eigenfunctions of the Laplace operator on bounded $N$-dimensional regions [2]. Our proofs rely mainly on the spectral theory of singular Sturm-Liouville systems.

We now present a more detailed mathematical formulation of the problem.

Let $\mathrm{S}-\mathrm{L}$ denote the singular Sturm-Liouville system

$$
u^{\prime \prime}(x, \lambda)-q(x) u(x, \lambda)=-\lambda u(x, \lambda)
$$

with boundary conditions

$$
u(0, \lambda) \cos \beta+u^{\prime}(0, \lambda) \sin \beta=0, \quad u(\infty, \lambda)<\infty,
$$

Received by the editors December 10, 1979; presented to the Society, January 3, 1980.

AMS (MOS) subject classifications (1970). Primary 34B25; Secondary 40A10, 42 A76.

Key words and phrases. Sturm-Liouville expansions, stable summability, singular.

${ }^{1}$ Research partially supported by the Resource Center for Science and Engineering, Atlanta University. 
where $q(x) \in L_{1}[0, \infty)$ is continuous, real valued, and $\beta \in[0,2 \pi]$. The functions $u(x, \lambda)$ (for all $\lambda$ in the spectrum) are normalized by the conditions

$$
u(0, \lambda)=\sin \beta, \quad u^{\prime}(0, \lambda)=-\cos \beta .
$$

The spectrum of S-L is bounded from below, discrete for $\lambda<0$, and continuous for $\lambda>0$ [3, Theorem 3.1, p. 209 and Theorem 3.2, p. 211]. The nonpositive spectrum is denoted by $\left\{\lambda_{n}\right\}=\Lambda_{1}$, and the associated eigenfunctions by $\left\{u\left(x, \lambda_{n}\right)\right\}$. Thus, $u(x, \lambda)$ denotes the eigenfunction associated with the spectral element $\lambda$, where $\lambda \in \Lambda=\Lambda_{1} \cup(0, \infty)$.

If $f \in L_{2}[0, \infty)$, the $\mathrm{S}-\mathrm{L}$ expansion of $f$ is given by

$$
\begin{aligned}
f(x) & \sim \sum_{\lambda_{n} \in \Lambda_{1}} F\left(\lambda_{n}\right) u\left(x, \lambda_{n}\right) d_{n}+\int_{0}^{\infty} F(\lambda) u(x, \lambda) d \rho(\lambda) \\
& =\int_{-b}^{\infty} F(\lambda) u(x, \lambda) d \rho(\lambda)
\end{aligned}
$$

where

$$
F(\lambda) \sim \int_{0}^{\infty} f(x) u(x, \lambda) d x
$$

In (4a), $-b=\inf (\lambda \in \Lambda), \rho(\lambda)$ is the spectral function of the system under the normalization (3), and $d_{n}=\rho\left(\lambda_{n}^{+}\right)-\rho\left(\lambda_{n}^{-}\right)$. In (4b), $F(\lambda)$ is the generalized Fourier transform of $f(x)$ (with respect to $\mathrm{S}-\mathrm{L}$ ) and $F \in L_{2, \rho}[-b, \infty)$, where $L_{2, \rho}$ denotes the square norm with respect to the measure $\rho(\lambda)$. Throughout this paper, the symbol $\sim$ denotes convergence in the $L_{2}$ norm as the upper limit of summation or integration becomes infinite.

Although the expansion in (4a) converges to $f(x)$ in the $L_{2}$ norm, it is well known that the convergence need not be pointwise. However, at sufficiently regular points of $f(x)$, an S-L expansion is summable to $f(x)$ using various summation methods [3], [6], [7]. These summation methods can be placed within a general class of methods, each of which is characterized by a summator function $\phi(t)$, as described below for the case of an S-L expansion.

Let $\phi(t):[-b, \infty) \rightarrow \mathbf{R}$ be bounded and continuous with $\phi(0)=1$. We denote the summability means, where they exist, of the S-L expansion (4a) by

$$
\begin{aligned}
S_{\alpha}(F ; x) & =\sum_{\lambda \in \Lambda_{1}} F\left(\lambda_{n}\right) \phi\left(\alpha \lambda_{n}\right) u\left(x, \lambda_{n}\right) d_{n}+\int_{0}^{\infty} F(\lambda) \phi(\alpha \lambda) u(x, \lambda) d \rho(\lambda) \\
& =\int_{-b}^{\infty} F(\lambda) \phi(\alpha \lambda) u(x, \lambda) d \rho(\lambda)
\end{aligned}
$$

where $\alpha$ is the summation parameter and $x$ is fixed. The $S-L$ expansion (4a) is called $\phi$-summable at $x_{0}$ if $\lim _{\alpha \rightarrow 0} S_{\alpha}\left(F ; x_{0}\right)$ exists and is called $\phi$-summable to $f$ at $x_{0}$ if $\lim _{\alpha \rightarrow 0} S_{\alpha}\left(F ; x_{0}\right)=f\left(x_{0}\right)$.

Let $\left\{F_{\gamma}(\lambda)\right\}_{\gamma}>0$ denote a generic net of approximations of $F(\lambda)$ such that corresponding to each value of the index $\gamma$ is an $L_{2, \rho}[b, \infty)$ function $F_{\gamma}(\lambda)$ satisfying $\left\|F_{\gamma}-F\right\|_{2, \rho} \leqslant \gamma$. We put

$$
F_{\gamma}(\lambda)=F(\lambda)+\Delta_{\gamma}(\lambda)
$$


and then have

$$
\left\|F_{\gamma}-F\right\|_{2, \rho}=\left\{\int_{-b}^{\infty}\left[\Delta_{\gamma}(\lambda)\right]^{2} d \rho(\lambda)\right\}^{1 / 2}<\gamma
$$

We now define a stable summation method (with respect to $\phi$ ) as a net $\left\{\mathscr{F}_{\gamma}\right\}_{\gamma>0}$ of functionals, $\mathscr{F}_{\gamma}: L_{2, \rho}[-b, \infty) \times[0, \infty) \rightarrow \mathbf{R}$, such that if $\lim _{\alpha \rightarrow 0} S_{\alpha}\left(F ; x_{0}\right)=f\left(x_{0}\right)$ then $\lim _{\gamma \rightarrow 0} \mathscr{F}_{\gamma}\left(F_{\gamma}, x_{0}\right)=f\left(x_{0}\right)$ holds for all $\left\{F_{\gamma}\right\}$. Thus a stable summation method associates with each value of the error index $\gamma$ a functional $\mathscr{F}_{\gamma}$ acting on S-L expansions, those functionals having the property that if $\gamma$ is small then $\mathscr{F}_{\gamma}\left(F_{\gamma} ; x_{0}\right)$ is close to the sum of the unperturbed S-L expansion $\phi$-summable to $f$ at $x_{0}$ and having coefficients $F(\lambda)$.

The main result of this paper, Theorem 1, says that the summability means $S_{\alpha}\left(F_{\gamma} ; x_{0}\right)$ furnish a stable summation method if the summation parameter $\alpha$ is appropriately scaled to go to zero with $\gamma$. More specifically, we shall prove that if $\int_{0}^{\infty} \phi^{2}(t) / t^{1 / 2} d t<\infty$ and $\alpha$ is scaled with $\gamma$ so that $\gamma / \alpha^{1 / 4} \rightarrow 0$ and $\alpha \rightarrow 0$ as $\gamma \rightarrow 0$, then $\mathscr{F}_{\gamma}=S_{\alpha}$ is a stable summation method. We will also show, in Theorem 2 , that this scaling is best possible, namely that if $\gamma / \alpha^{1 / 4} \nrightarrow 0$ as $\gamma \rightarrow 0$ then $S_{\alpha}$ is not a stable summation method. Krukovskii [2] has obtained an analogous sharp result for expansions in eigenfunctions of the Laplace operator in arbitrary bounded $N$-dimensional regions.

2. Proofs of Theorems 1 and 2. We will require the following well-known lemma.

LEMMA 1. As $\lambda \rightarrow \infty$, the eigenfunctions $u(x, \lambda)$ of $S-L$ satisfy

(a) $u(x, \lambda)=-\sin \sqrt{\lambda} x \cos \beta / \sqrt{\lambda}+O(1 / \lambda)$ if $\sin \beta=0$,

(b) $u(x, \lambda)=\cos \sqrt{\lambda} x \sin \beta+O(1 / \sqrt{\lambda})$ if $\sin \beta \neq 0$,

uniformly in $x \in[0, \infty)$.

Proof. For $\lambda>0, u(x, \lambda)$ satisfies

$$
\begin{aligned}
u(x, \lambda)= & \cos \sqrt{\lambda} x \sin \beta-\frac{\sin \sqrt{\lambda} x \cos \beta}{\sqrt{\lambda}} \\
& +\frac{1}{\sqrt{\lambda}} \int_{0}^{x} \sin [\sqrt{\lambda}(x-t)] q(t) u(t, \lambda) d t
\end{aligned}
$$

[3, Equation 3.5, p. 205], and the proof follows using $q(t) \in L_{1}$ and $u(t, \lambda)$ bounded in $t$ for each $\lambda$.

We can approximate the spectral function $\rho(\lambda)$ for large $\lambda$ using the following lemma.

LEMMA 2. As $\lambda \rightarrow \infty$

$$
\begin{aligned}
& \rho^{\prime}(\lambda)=\frac{\sqrt{\lambda}}{\pi \cos ^{2} \beta}\left[1+O\left(\frac{1}{\sqrt{\lambda}}\right)\right]=O(\sqrt{\lambda}), \quad \text { if } \sin \beta=0 \\
& \rho^{\prime}(\lambda)=\frac{1}{\pi \sqrt{\lambda} \sin ^{2} \beta}\left[1+O\left(\frac{1}{\sqrt{\lambda}}\right)\right]=O\left(\frac{1}{\sqrt{\lambda}}\right), \quad \text { if } \sin \beta \neq 0 .
\end{aligned}
$$


Proof. Using the notation of $[3$, p. 206] define

$$
\begin{aligned}
& \mu(\lambda)=\sin \beta-\frac{1}{\sqrt{\lambda}} \int_{0}^{\infty} \sin (\sqrt{\lambda} t) q(t) u(t, \lambda) d t, \\
& \nu(\lambda)=\frac{-\cos \beta}{\sqrt{\lambda}}+\frac{1}{\sqrt{\lambda}} \int_{0}^{\infty} \cos (\sqrt{\lambda} t) q(t) u(t, \lambda) d t .
\end{aligned}
$$

Using Lemma 1, we obtain $\mu(\lambda)=O(1 / \lambda)$ and $\nu(\lambda)=-\cos \beta / \sqrt{\lambda}+O(1 / \lambda)$ if $\sin \beta=0 ; \mu(\lambda)=\sin \beta+O(1 / \sqrt{\lambda})$ and $\nu(\lambda)=O(1 / \sqrt{\lambda})$ if $\sin \beta \neq 0$. From [3, Theorem 3.2, p. 211] we have

$$
\rho^{\prime}(\lambda)=\frac{1}{\pi} \cdot \frac{1}{\sqrt{\lambda}}\left[\frac{1}{\mu^{2}(\lambda)+\nu^{2}(\lambda)}\right]
$$

and the lemma follows upon substitution of the above estimates for $\mu$ and $\nu$.

THEOREM 1. Suppose the following conditions hold.

(a) $\int_{0}^{\infty}\left(\phi^{2}(\lambda) d \lambda / \lambda^{1 / 2}\right)<\infty$,

(b) $S_{\alpha}(F ; x) \rightarrow f(x)$ as $\alpha \rightarrow 0$ uniformly on a bounded subset $E$ of $[0, \infty)$,

(c) $\alpha$ is a function of $\gamma$ such that as $\gamma \rightarrow 0, \alpha \rightarrow 0$ and $\gamma / \alpha^{1 / 4} \rightarrow 0$.

Then $S_{\alpha}\left(F_{\gamma} ; x\right) \rightarrow f(x)$ as $\gamma \rightarrow 0$ uniformly on $E$; in particular, $S_{\alpha}$ is a stable summation method.

Proof. As $\left|f(x)-S_{\alpha}\left(F_{\gamma} ; x\right)\right| \leqslant\left|f(x)-S_{\alpha}(F ; x)\right|+\left|S_{\alpha}\left(F-F_{\gamma} ; x\right)\right|$, it suffices to show that $S_{\alpha}\left(F-F_{\gamma} ; x\right) \rightarrow 0$ uniformly on $E$.

We have $\left|S_{\alpha}\left(F-F_{\gamma} ; x\right)\right|=\left|\int_{0}^{\infty} \Delta_{\gamma}(\lambda) \phi(\alpha \lambda) u(x, \lambda) d \rho(\lambda)\right|$ where $\Delta_{\gamma}$ satisfies (6) and (7). By the Cauchy-Schwarz inequality and (7)

$$
\left|S_{\alpha}\left(F-F_{\gamma} ; x\right)\right| \leqslant \gamma\left[\int_{-b}^{\infty} \phi^{2}(\alpha \lambda) u^{2}(x, \lambda) d \rho(\lambda)\right]^{1 / 2} .
$$

We show first that for $a>-b$, the function $\int_{-b}^{a} \phi(\alpha \lambda) u^{2}(x, \lambda) d \rho(\lambda)$ is uniformly bounded for $x \in E$ and $\alpha>0$. Let $K \supset E$ be a compact set. In the compact rectangle $K \times[-b, a]$ of the $(x, \lambda)$ plane, the solutions of (1) satisfying initial conditions (3) are jointly continuous in $x$ and $\lambda$ by a well-known result of differential equations [1, p. 145]. Thus the $u(x, \lambda)$, which satisfy (1), (2) and (3) must be uniformly bounded on $E \times[-b, a]$. By assumption, $\phi^{2}(\alpha \lambda)$ is bounded. We have then, for appropriately chosen constants $C_{1}$ and $C_{2}$,

$$
\int_{-b}^{a} \phi^{2}(\alpha \lambda) u^{2}(x, \lambda) d \rho(\lambda) \leqslant C_{1} \int_{-b}^{a} d \rho(\lambda)=C_{1}(\rho(a)-\rho(-b)) \leqslant C_{2}<\infty .
$$

We now estimate $\int_{a}^{\infty} \phi^{2}(\alpha \lambda) u^{2}(x, \lambda) d \rho(\lambda)$, considering the cases $\sin \beta=0$ and $\sin \beta \neq 0$.

(a) $\sin \beta=0$. Using Lemma 1 , let $a$ be sufficiently large so that $|u(x, \lambda)|<2 / \sqrt{\lambda}$ and $\rho^{\prime}(\lambda) \leqslant C_{3} \sqrt{\lambda}$ for $\lambda \geqslant a$ and $x \in[0, \infty)$. Then

$$
\int_{a}^{\infty} \phi^{2}(\alpha \lambda) u^{2}(x, \lambda) d \rho(\lambda) \leqslant 4 C_{3} \int_{0}^{\infty} \frac{\phi^{2}(\alpha \lambda)}{\sqrt{\lambda}} d \lambda=\frac{4 C_{3}}{\sqrt{\alpha}} \int_{0}^{\infty} \frac{\phi^{2}(w)}{\sqrt{w}} d w=\frac{C_{4}}{\sqrt{\alpha}}
$$


(b) $\sin \beta \neq 0$. We now let $a$ be sufficiently large so that $u(x, \lambda)<2$ and $\rho^{\prime}(\lambda)<C_{5} / \sqrt{\lambda}$ for $\lambda \geqslant a$ and $x \in[0, \infty)$. Then

$$
\int_{a}^{\infty} \phi^{2}(\alpha \lambda) u^{2}(x, \lambda) d \rho(\lambda)<4 C_{5} \int_{0}^{\infty} \frac{\phi^{2}(\alpha \lambda)}{\sqrt{\lambda}}<\frac{C_{6}}{\sqrt{\alpha}} .
$$

Using (9)-(11) in (8) shows that $\left|S_{\alpha}\left(F-F_{\gamma} ; x\right)\right|<\gamma\left[C_{2}+C_{7} / \sqrt{\alpha}\right]^{1 / 2}<$ $\gamma C_{8} / \alpha^{1 / 4} \rightarrow 0$ as $\gamma \rightarrow 0$, proving the theorem.

The following theorem shows that the scaling $\gamma / \alpha^{1 / 4} \rightarrow 0$ is best possible.

TheOREM 2. If $\phi(t)$ satisfies $\int_{0}^{\infty} \phi^{2}(\lambda) / \sqrt{\lambda} d \lambda<\infty$ and $\gamma / \alpha^{1 / 4} \nrightarrow 0$ as $\gamma \rightarrow 0$ then $S_{\alpha}$ is not a stable summation method for $x_{0} \in(0, \infty)$.

Proof. To prove Theorem 2 we will explicitly produce $\left\{F_{\gamma}(\lambda)\right\}$ such that $S_{\alpha}\left(F-F_{\gamma} ; x_{0}\right) \nrightarrow 0$. We assume that $\alpha$ is a specified function of $\gamma$ such that $\gamma \rightarrow 0$ and $\gamma / \alpha^{1 / 4} \nrightarrow 0$. If $\alpha(\gamma) \neq 0$, pick $F_{\gamma}(\lambda)$ so that

$$
F_{\gamma}(\lambda)-F(\lambda)=\Delta_{\gamma}(\lambda)=\gamma \frac{\phi(\alpha \lambda) u\left(x_{0}, \lambda\right)}{\left\|\phi(\alpha \lambda) u\left(x_{0}, \lambda\right)\right\|_{2, \rho}} .
$$

The computations of Theorem 1 show that if $\alpha \neq 0$ then $\Delta_{\gamma}(\lambda) \in L_{2, \rho}$ and therefore so is $F_{\gamma}$. The case of $\alpha(\gamma)=0$ will require a different definition of $F_{\gamma}$ to be given later.

We have from (12), $\left\|\Delta_{\gamma}\right\|_{2, \rho}=\gamma$. Applying the definition of $S_{\alpha}$, we have

$$
\left|S_{\alpha}\left(F-F_{\gamma} ; x_{0}\right)\right|=\gamma\left\|\phi(\alpha \lambda) u\left(x_{0}, \lambda\right)\right\|_{2, \rho}>\gamma\left[\int_{a}^{\infty} \phi^{2}(\alpha \lambda) u^{2}\left(x_{0}, \lambda\right) d \rho(\lambda)\right]^{1 / 2}
$$

for any $a>-b$. Estimating (13) we again consider the two cases $\sin \beta=0$ and $\sin \beta \neq 0$.

(a) $\sin \beta=0$. Using Lemmas 1 and 2 pick $a>0$ large enough so that for an appropriately chosen constant $C_{1}$,

$$
u^{2}(x, \lambda) \geqslant \frac{\sin ^{2} \sqrt{\lambda} x}{\lambda}-\frac{C_{1}}{\lambda^{3 / 2}}, \quad \rho^{\prime}(\lambda)>\frac{\sqrt{\lambda}}{4} \quad \text { for } \lambda>a .
$$

Next, choose $C_{2}>0$ so that $\phi^{2}(x) \geqslant \frac{1}{2}$ for $0<x<C_{2}$. We then have for small $\alpha$,

$$
\begin{aligned}
\int_{a}^{\infty} \phi^{2}(\alpha \lambda) u^{2}(x, \lambda) d \rho(\lambda) & >\frac{1}{2} \int_{a}^{C_{2} / \alpha} u^{2}(x, \lambda) d \rho(\lambda) \\
& \geqslant \frac{1}{4 \sqrt{\alpha}} \int_{\sqrt{\alpha a}}^{\sqrt{C_{2}}}\left[\sin ^{2} \frac{x_{0}}{\sqrt{\alpha}} w-\frac{\sqrt{\alpha} C_{1}}{w}\right] d w
\end{aligned}
$$

where, to obtain (15), we have applied the inequalities (14) and the substitution $\alpha \lambda=w^{2}$. For $\alpha$ sufficiently small $\int_{\sqrt{\alpha a}}^{\sqrt{C_{2}}} \sin ^{2}\left(x_{0} / \sqrt{\alpha}\right) w d w>C_{3}$ while

$$
\sqrt{\alpha} C_{1} \int_{\sqrt{\alpha a}}^{\sqrt{C_{2}}} d w / w<C_{3} / 2
$$

for $C_{3}$ appropriately chosen. We then have from (15), $\int_{a}^{\infty} \phi^{2}(\alpha \lambda) u^{2}(x, \lambda) d \rho(\lambda)>$ $C_{3} / 8 \sqrt{\alpha}$ and finally

$$
\left|S_{\alpha}\left(F-F_{\gamma} ; x_{0}\right)\right|>\left(\gamma / \alpha^{1 / 4}\right) C_{4}
$$


(b) $\sin \beta \neq 0$. An argument analogous to that of the case $\sin \beta=0$ leads to the desired inequality of the form (16).

To complete the proof we need only specify appropriate $F_{\gamma}$ when $\alpha(\gamma)=0$. In this case we put $\alpha^{*}=\gamma^{4}, M=\sup |\phi(x)|$ and

$$
F_{\gamma}-F=\Delta_{\gamma}(\lambda)=\frac{\gamma}{M} \frac{\phi^{2}\left(\alpha^{*} \lambda\right) u\left(x_{0}, \lambda\right)}{\left\|\phi\left(\alpha^{*} \lambda\right) u\left(x_{0}, \lambda\right)\right\|_{2, \rho}} .
$$

Then $\left\|\Delta_{\gamma}(\lambda)\right\|_{2, \rho} \leqslant \gamma,\left|S_{\alpha}\left(F-F_{\gamma} ; x_{0}\right)\right|=(\gamma / M)\left\|\phi\left(\alpha^{*} \lambda\right) u\left(x_{0}, \lambda\right)\right\|_{2, \rho}$ and we obtain, analogous to (16), $\left|S_{\alpha}\left(F-F_{\gamma} ; x_{0}\right)\right| \geqslant(\gamma / M)\left(C_{3} /\left(\alpha^{*}\right)^{1 / 4}\right)=C_{3} / M$. Thus, for all $\gamma$ sufficiently small we have

$$
\left|S_{\alpha}\left(F-F_{\gamma} ; x_{0}\right)\right| \geqslant C_{3} \min \left(1 / M, \gamma / \alpha^{1 / 4}\right) \nrightarrow 0 \quad \text { as } \alpha \rightarrow 0
$$

and the theorem is proved.

REMARK. The case $q(x)=0, \beta=\pi, u(x, \lambda)=\sin \sqrt{\lambda} x / \sqrt{\lambda}, b=0$, shows that Theorem 2 need not hold for $x_{0}=0$, since in this case $u(0, \lambda)=0$ for all $\lambda \in[-b, \infty)$.

3. Applications. The practical application of Theorem 1 requires the a priori $\phi$-summability of an S-L expansion (4). In this section we discuss two summation methods for which such results are known.

(a) Riesz summability. The Riesz summation method uses the summator function $\phi(t)=\{1-t, t<1 ; 0, t \geqslant 1\}$. Riesz summability is used in spectral theory associated with linear ordinary differential equations. It is a natural tool for proving theorems concerning equisummability and equiconvergence for expansions in the eigenfunctions of a Sturm-Liouville operator (see [3, Chapters 7 and 8]).

It is well known [5] that the S-L eigenfunction expansion of $f \in L_{2}[0, \infty)$ is Riesz summable pointwise to $f$ at Lebesgue points, that is, points $x$ at which

$$
\int_{-\eta}^{\eta}|f(x+\eta)-f(x)| d x=o(\eta), \quad \eta \rightarrow 0 .
$$

We may combine this fact with Theorems 1 and 2 to obtain

Theorem 3. For the Riesz summation method, $\phi(t)=(1-t, t \leqslant 1 ; 0, t>1)$ :

(i) If, as $\gamma \rightarrow 0, \alpha \rightarrow 0$ and $\gamma / \alpha^{1 / 4} \rightarrow 0$; and $x_{0}>0$ is a Lebesgue point of the $L_{2}[0, \infty)$ function $f$ then $S_{\alpha}\left(F_{\gamma} ; x_{0}\right) \rightarrow f\left(x_{0}\right)$ as $\gamma \rightarrow 0$ for all $\left\{F_{\gamma}\right\}$.

(ii) If $\gamma \rightarrow 0$ but $\gamma / \alpha^{1 / 4} \nrightarrow 0$ then for any $x_{0} \in(0, \infty), S_{\alpha}\left(F_{\gamma}, x_{0}\right) \nrightarrow f\left(x_{0}\right)$ for suitably chosen $\left\{F_{\gamma}\right\}$.

Proof. Part (i) follows easily since $\phi(t)$ satisfies the integral condition of Theorems 1 and 2. As for part (ii), if $S_{\alpha}\left(F, x_{0}\right) \nrightarrow 0$ as $\alpha \rightarrow 0$, choose $F_{\gamma}=F$; otherwise Theorem 2 applies.

(b) Stieltjes summability. The Stieltjes summation method uses the summator function $\phi(t)=1 /(1+t)$. Its application to the summation of integrals, and in particular the S-L expansion (4), has been studied in [4]. Prior examples of its use in summing discrete eigenfunction expansions occur in [5] and [6].

The Sturm-Liouville system considered in [4] is a special case of S-L. Accordingly, we define the system S-L*: 
$u^{\prime \prime}(x, \lambda)-q(x) u(x, \lambda)=-\lambda u(x, \lambda), \quad u(0, \lambda)=0, u^{\prime}(0, \lambda)=1, u(\infty, \lambda)<\infty$, where $q(x) \in L_{1}[0, \infty)$ is bounded and continuous.

The following theorem is proved in [4].

THEOREM 4. For the Stieltjes summation method applied to $S$ - $L^{*}$ eigenfunction expansions, if $x_{0}>0$ is a continuity point of $f \in L_{2}[0, \infty)$ and $\alpha=k \gamma^{2}$ for some $k>0$, then $S_{\alpha}\left(F_{\gamma} ; x_{0}\right) \rightarrow f\left(x_{0}\right)$ as $\gamma \rightarrow 0$.

Since Theorem 4 implies, in particular, that $S_{\alpha}\left(F ; x_{0}\right) \rightarrow f\left(x_{0}\right)$, we may apply Theorems 1 and 2 to show that the conclusion of Theorem 4 holds for the sharp scaling requirement of $\alpha \rightarrow 0$ and $\gamma / \alpha^{1 / 4} \rightarrow 0$ as $\gamma \rightarrow 0$. If we assume that $f$ is bounded in addition to being in $L_{2}[0, \infty)$, Theorem 4 can be further extended to include Lebesgue points of $f$. We shall prove this next, using results and notation from [4].

Let $G_{q}(x, s)$ denote the Green's function for the differential equation $-u^{\prime \prime}+$ $q(x) u=0$ with boundary conditions $u(0)=0, u^{\prime}(0)=1$, where $q(x) \in L_{1}[0, \infty)$ is bounded and continuous.

LEMMA 3. If $x-\eta>0$ and $\alpha>0$, the following hold as $\alpha, \eta \rightarrow 0$.

(a) $\int_{x-\eta}^{x+\eta} G_{q+1 / \alpha}(x, s) d s=\alpha(1+O(\alpha))\left[1+O\left(e^{-\eta / 2 \sqrt{\alpha}}\right)\right]$,

(b) $\int_{0}^{\infty} G_{q+1 / \alpha}^{2}(x, s) d s=O\left(\alpha^{3 / 2}\right)$,

(c) $\int_{K} G_{q+1 / \alpha}(x, s) d s=\alpha O\left(e^{-\eta / \sqrt{\alpha}}\right)$ where $K=(0, x-\eta) \cup(x+\eta, \infty)$,

(d) $\sup _{s>0} G_{q+1 / \alpha}(x, s)=O(\sqrt{\alpha})$.

Proof. The proofs of statements (a), (b) and (d) are in [4]; the proof of (c) follows from the explicit bounds on $G_{q+1 / \alpha}(x, s)$ given in [4].

LEMMA 4. Let $f \in L_{2}[0, \infty)$ be real valued. If $f$ is bounded and $x_{0}>0$ is a Lebesgue point of $f$, then $S_{\alpha}\left(F ; x_{0}\right) \rightarrow f\left(x_{0}\right)$ as $\alpha \rightarrow 0$.

Proof. In [4], it is shown that

$$
S_{\alpha}\left(F ; x_{0}\right)=\frac{1}{\alpha} \int_{0}^{\infty} G_{q+1 / \alpha}\left(x_{0}, s\right) f(s) d s .
$$

Now, for $\eta>0$, and $x-\eta>0$, by (17)

$$
\begin{aligned}
S_{\alpha}\left(F ; x_{0}\right)-f\left(x_{0}\right)= & \frac{1}{\alpha} \int_{x_{0}-\eta}^{x_{0}+\eta} G_{q+1 / \alpha}\left(x_{0}, s\right) f\left(x_{0}\right) d s-f\left(x_{0}\right) \\
& +\frac{1}{\alpha} \int_{x_{0}-\eta}^{x_{0}+\eta} G_{q+1 / \alpha}\left(x_{0}, s\right)\left[f(s)-f\left(x_{0}\right)\right] d s \\
& +\frac{1}{\alpha} \int_{K} G_{q+1 / \alpha}\left(x_{0}, s\right) f(s) d s
\end{aligned}
$$

where $K$ is as in Lemma 3. Now, by Lemma 3

$$
\frac{1}{\alpha} \int_{K} G_{q+1 / \alpha}\left(x_{0}, s\right) f\left(x_{0}\right) d s=f\left(x_{0}\right)\left[1+O(\alpha)+O\left(e^{-\eta / 2 \sqrt{\alpha}}\right)\right] \quad(\alpha, \eta \rightarrow 0) .
$$


Also,

$$
\begin{aligned}
\frac{1}{\alpha} \int_{x_{0}-\eta}^{x_{0}+\eta}\left|G_{q+1 / \alpha}\left(x_{0}, s\right)\left[f(s)-f\left(x_{0}\right)\right]\right| d s & \leqslant O\left(\frac{1}{\sqrt{\alpha}}\right) \int_{x_{0}-\eta}^{x_{0}+\eta}\left|f(s)-f\left(x_{0}\right)\right| d s \\
& =\frac{o(\eta)}{\sqrt{\alpha}}
\end{aligned}
$$

and

$$
\left|\frac{1}{\alpha} \int_{K} G_{q+1 / \alpha}\left(x_{0}, s\right) f(s) d s\right| \leqslant \frac{\|f\|_{\infty}}{\alpha} \int_{K} G_{q+1 / \alpha}\left(x_{0}, s\right) d s=O\left(e^{-\eta / \sqrt{\alpha}}\right) .
$$

Choose $\eta(\alpha)$ so that as $\alpha \rightarrow 0, \eta / \sqrt{\alpha} \rightarrow \infty$ and $o(\eta) / \sqrt{\alpha} \rightarrow 0$. For such a choice of $\eta, S_{\alpha}\left(F ; x_{0}\right) \rightarrow f\left(x_{0}\right)$ by (18a)-(18c). This proves the Stieltjes summability of $f$ at the Lebesgue point $x_{0}$.

Using Theorems 1, 2, 4 and Lemma 4, we can write the following result for the stable Stieltjes summability of S-L* eigenfunction expansions.

TheORem 5. Let $f \in L_{2}[0, \infty)$, and $x_{0}>0$. Then

(i) if $x_{0}$ is a continuity point of $f$, or

(ii) $f$ is bounded and $x_{0}$ is a Lebesgue point of $f$

and if $\gamma / \alpha^{1 / 4} \rightarrow 0$ and $\alpha \rightarrow 0$ as $\gamma \rightarrow 0$ we have $S_{\alpha}\left(F_{\gamma} ; x_{0}\right) \rightarrow f\left(x_{0}\right)$ as $\gamma \rightarrow 0$. If (i) or (ii) holds but $\gamma / \alpha^{1 / 4} \nrightarrow 0$ as $\gamma \rightarrow 0$ then there exists $\left\{F_{\gamma}\right\}$ such that $S_{\alpha}\left(F_{\gamma} ; x_{0}\right) \nrightarrow$ $f\left(x_{0}\right)$ as $\gamma \rightarrow 0$.

Proof. The summator function $\phi(t)=1 /(1+t)$ satisfies the integral condition of Theorem 1. The proof then follows upon application of Theorems 1, 2, 4 and Lemma 4.

Theorems analogous to Theorems 3 and 5 can be obtained for other summability methods, providing that $\phi(t)$ satisfies the integral condition of Theorem 1 and the unperturbed eigenfunction expansions are known a priori to be $\phi$-summable.

\section{REFERENCES}

1. G. Birkhoff and G.-C. Rota, Ordinary differential equations, 3rd ed., Wiley, New York, 1978.

2. N. M. Krukovskii, On the Tikhonov-stable summation of Fourier series with perturbed coefficients by some regular methods, Moscow Univ. Math. Bull. (3) 28 (1973), 17-23.

3. B. M. Levitan and I. S. Sargsjan, Introduction to spectral theory: selfadjoint ordinary differential operators, Math. Monos., vol. 39, Amer. Math. Soc., Providence, R. I., 1975.

4. L. A. Raphael, A generalized Stieltjes transform summability method and summing Sturm-Liouville expansions, SIAM J. Math. Anal. (to appear).

5. A. N. Tihonov, Stable methods for the summation of Fourier series, Soviet Math. Dokl. 5 (1964), 641-644

6. E. C. Titchmarsh, Eigenfunction expansions. I, 2nd ed., Oxford Univ. Press, Oxford, 1962.

7. A. Zygmund, Trigonometric series, Cambridge Univ. Press, Cambridge, 1959.

Department of Mathematics, West Virginia University, Morgantown, West Virginia 26506

Department of Mathematics, Boston University, Boston, Massachusetts 02215

Department of Mathematics, Clark College, Atlanta, Georgia 30314 